\title{
Proton decay matrix elements with physical quark
} masses

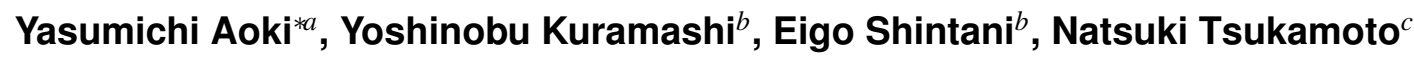 (PACS Collaboration)}

${ }^{a}$ RIKEN Center for Computational Science, Kobe 650-0047, Japan

${ }^{b}$ Center for Computational Sciences, University of Tsukuba, Tsukuba 305-8577, Japan

${ }^{c}$ Department of Physics, Tohoku University, Sendai 980-8578, Japan

E-mail: yasumichi.aoki@riken.jp

\begin{abstract}
Proton decay matrix elements in QCD are indispensable quantities to constrain GUT models through the lower bound of the proton lifetime measured in the current and future underground experiments. Results obtained with lattice QCD so far have potentially largest systematic uncertainty on the chiral extrapolation. We report on the relevant form factors of one of the dominant decay processes: a proton decaying into a pion and an anti-lepton with a three-quark operator, using the physical-point, improved Wilson-fermion configurations generated by the PACS collaboration at the lattice spacing of $0.084 \mathrm{fm}$.
\end{abstract}

37th International Symposium on Lattice Field Theory - Lattice2019

16-22 June 2019

Wuhan, China

${ }^{*}$ Speaker. 


\section{Introduction}

Proton decay is a smoking-gun signal of the physics beyond the standard model. With no signal of the decay, though, the lower limit of the proton lifetime is prolonged, and now it counts $1.6 \times 10^{34}$ years (90\% confidence level) [1] for the partial lifetime of proton decaying into a neutral pion and a positron. The partial lifetime limit is being extended with the current experiments and will reach further in the planned future experiments such as DUNE and HyperKamiokande.

The grand unified theories (GUTs) are the origin of the decay as the baryon number is not protected in such theories. To get information of the theory with the experimental lifetime bounds, one needs to know the related low energy QCD matrix elements with proton initial and meson final states. These are the proton decay matrix elements computed in this study.

Most recent lattice computation of the proton decay matrix elements uses $N_{f}=2+1$ domainwall fermions [2]. Chiral extrapolation in the average $\hat{m}$ of the physical $u$ and $d$ quark masses is performed from four $\hat{m}$ values corresponding to the pion mass $340-700 \mathrm{MeV}$. Although the systematic error from the long chiral extrapolation is attempted to be estimated, it is within the polynomial extrapolation. If large non-analytic behavior is present through the physical point towards the chiral limit, ${ }^{1}$ that will leave the current error estimate insufficient.

Here we report on our attempt to compute the proton decay matrix elements at the physical quark masses ( $\hat{m}$ and strange quark mass) to remove the systematic error of the chiral extrapolation completely. ${ }^{2}$ All the results reported in this study are preliminary.

\section{Lattice parameters and measurement procedures}

We use, in this study, the $N_{f}=2+1$ physical point ensemble generated by the PACS collaboration with Iwasaki gauge $(\beta=1.82)$ and tree-level improved Wilson fermion with the stout link ( $\rho=0.1, N=6$ ), corresponding to the lattice cutoff of $a^{-1}=2.233(18) \mathrm{GeV}$. The lattice volume $64^{4}$ is mostly used while $96^{4}$ is used for a check of the finite volume effect.

A simplified version of the all mode averaging (AMA) [5, 6] is used here: for each lattice one quark propagator with a stringent stopping condition is combied with many propagators with a sloppy solver. A typical number of the sloppy solve for the low energy matrix elements are 256 for each lattice. About 100 lattices are used to calculate hadronic two- and three-point functions for the proton decay with gauge covariant Gaussian smearing for quarks in the hadron interpolating operators to enhance the ground state signals. For the non-perturbative renormalization, only one point-source propagator is calculated for each lattice.

\section{RI/SMOM non-perturbative renormalization}

RI/MOM scheme [7] is defined at the chiral limit where all the quark mass vanishes. With the physical point ensemble alone for $N_{f}=2+1$, the results may suffer from the non-zero quark mass, especially for strange quark. RI/SMOM schemes [8] are also defined at the chiral limit. However, due to the use of non-exceptional momenta the the effect of the low scale like light quark mass

\footnotetext{
${ }^{1}$ which includes the one due to the topological stability of the proton state within the chiral Skyrme bag model [3]

${ }^{2}$ There is a similar on-going activity using domain-wall fermions [4].
} 
is much reduced compared to the RI/MOM scheme (see, e.g. [9]), which is usually defined with the exceptional momenta. The original RI/MOM scheme formulation for the proton-decay, threequark operator [10] uses non-exceptional momenta for the three-quark vertex functions, combined with the quark wavefunction renormalization determined from the axial vector vertex with the exceptional momentum configuration. Here we use the non-exceptional momenta, namely the symmetric subtraction point for the bilinear quark vertex as well, which minimizes the systematic effect of non-zero quark mass throughout the whole renormalization program.

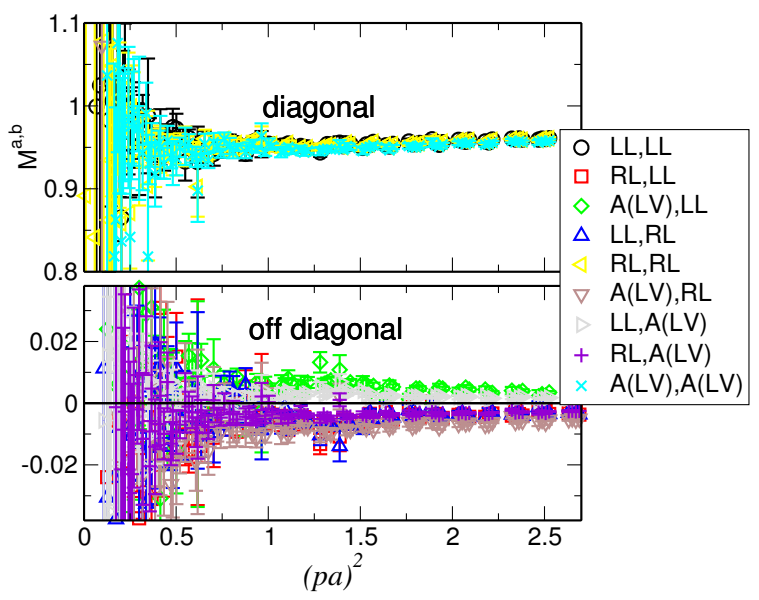

Figure 1: Projected vertex functions (see [10] for details) with a non-exceptional momentum configuration in RI/MOM scheme as functions of momentum squared in lattice units.

Figure 1 shows projected vertex functions with the procedure described in [10] as a function of injected momentum squared. The $64^{4}$ PACS ensemble is used. The diagonal elements of this $3 \times 3$ matrix $M^{a, b}$ are shown in the upper panel and the off-diagonal elements in the lower. The size of the off-diagonal elements for $(p a)^{2} \gtrsim 0.7$, the momentum range to be used in the next process, is as small as $1 \%$ of the diagonal. This indicates that the off-diagonal elements of the renormalization matrix $Z^{a, b}$, that express the mixing coefficients, are as small as $1 \%$ of the diagonal, since $M^{a, b}$ is proportional to the inverse of $Z_{a, b}$. One can safely neglect the effect of the off-diagonal elements, since we have much larger errors from the other sources as we will see below. As such the nucleon decay operators are treated as multiplicatively renormalized.

The renormalization factor of an RI scheme, for example for $\Gamma=L L$ operator, is then given by $Z_{L L}=Z_{q}^{3 / 2} / M^{L L, L L}$. The wavefunction renormalization is canceled by taking a ratio with respect to the vertex function of the local (axial) vector current: $\Lambda_{A}^{3 / 2} / M^{L L, L L}=Z_{L L} / Z_{A}^{3 / 2}$ or $\Lambda_{V}^{3 / 2} / M^{L L, L L}=$ $Z_{L L} / Z_{V}^{3 / 2}$. The left hand side is all evaluated within RI schemes. Supplying the value of $Z_{A}$ or $Z_{V}$ will give an estimate of $Z_{L L}$. Here we use the estimates of $Z_{A}$ and $Z_{V}$ with the Schrödinger functional scheme [11]. The results so obtained for RI/SMOM and RI/SMOM $\gamma_{\mu}$ schemes for each momentum values are first averaged among same $(p a)^{2}$ value, matched to $\overline{\mathrm{MS}}$ scheme through oneloop perturbation theory with $\mu=\sqrt{p^{2}}$, and then ran to $\mu=2 \mathrm{GeV}$ with two-loop. The obtained results for the four intermediate SMOM schemes are plotted in Fig. 2 (a) as functions of matching scale squared.

Ideally there should not be no matching point momentum dependence if there is neither sys- 

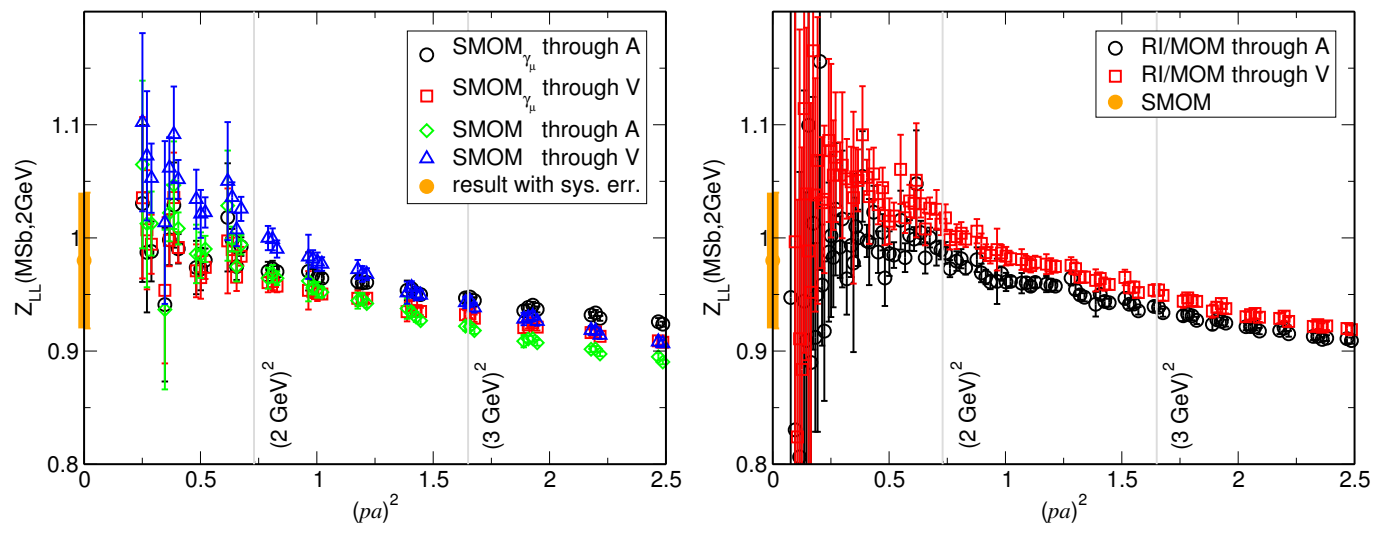

Figure 2: (a) (left panel) Renormalization factor of $L L$ operator in $\overline{\mathrm{MS}} \mathrm{NDR}$ at $\mu=2 \mathrm{GeV}$ as a function of matching scale squared. Results from four intermediate RI/SMOM matching schemes are plotted. Orange bar at $(p a)^{2}=0$ indicates the representative central value with total error, which is explained in detail in the text. (b) (right panel) same as (a) but, with RI/MOM intermediate matching scheme. The orange bar at $(p a)^{2}=0$ is taken from (a), which is determined from the RI/SMOM intermediate schemes for comparison.

tematic error due to the use of perturbation theory (PT) for the matching and running, nor the one due to the lattice discretization error $O\left((p a)^{2}\right)$. The systematic errors from the use of PT are of the truncation error and the contamination of the spontaneous or explicit (predominantly chiral) symmetry breaking to the NPR data, which are not taken into account in PT. The $(p a)^{2}$ dependence and the scatter among different intermediate schemes are mixture of these effect. Among them the leading discretization error will vanish as $(p a)^{2}$ tends to zero, while the effect of spontaneous chiral symmetry breaking will grow. Therefore these effects are hard to disentangle. In this situation we estimate the central value of the renormalization factor and its error by taking into account by fist selecting representative values as many as possible and then examine their scatter. First we set the prime window of the data to be $(2 \mathrm{GeV})^{2}<p^{2}<(3 \mathrm{GeV})^{2}$. This is to avoid the use of data potentially heavily contaminated by the symmetry breaking effect or large discretization error. We can pick any value in this window as a representative. We can also take $(p a)^{2} \rightarrow 0$ extrapolated result for each intermediate scheme. Maximum and minimum of these representative values are taken and then the central value is defined as the average of two and the error is the half stretch from minimum to maximum. The statistical error is neglected since it is much smaller than this systematic error.

Figure 2 (b) shows the same quantity but obtained with the conventional RI/MOM intermediate schemes, which suffer from larger systematic error from chiral symmetry breaking and strange quark mass. One see reasonable consistency to the result from SMOM schemes indicated as orange bar at $(p a)^{2}=0$.

The same procedure is followed for $R L$ operator as well and we get the renormalization factors,

$$
Z_{L L}^{\overline{\mathrm{MS}}}(\mu=2 \mathrm{GeV})=0.98(6), Z_{R L}^{\overline{\mathrm{MS}}}(\mu=2 \mathrm{GeV})=0.98(7) .
$$

Note that the factor is consistent with 1 within the error. In the following sections we present the preliminary results of the bare matrix elements, which can be regarded as those renormalized in $\overline{\mathrm{MS}} \mathrm{NDR}$ at $\mu=2 \mathrm{GeV}$ by $6-7 \%$ accuracy. 


\section{Proton decay matrix elements}

The proton decay matrix element for an initial nucleon with momentum $k$ and spin $s$ to a final pseudoscalar with momentum $p$ reads [12]

$$
\left\langle P(p)\left|\mathscr{O}^{I}\right| N(k, s)\right\rangle=P_{\Gamma^{I}}\left[W_{0}^{I}\left(q^{2}\right)-\frac{i \phi}{m_{N}} W_{1}^{I}\left(q^{2}\right)\right] u_{N}(k, s),
$$

with $\mathscr{O}^{I}$ being a baryon-number violating three-quark operator with a certain flavor structure as well as with inherent internal $\Gamma^{I}$ and external $\Gamma^{I}$ structures taking either left-handed $\left(P_{L}\right)$ or righthanded $\left(P_{R}\right)$ projection. These variations are expressed in a single label $I . W_{0}^{I}\left(W_{1}^{I}\right)$ is the relevant (irrelevant) form factor, depending on the squared momentum transfer $q^{2}$ where $q=k-p . m_{N}$ and $u_{N}$ are the mass and spinor of the nucleon. Neglecting $O\left(m_{l} / m_{N}\right)$ where $m_{l}$ is the mass of the outgoing anti-lepton, the partial width is given by

$$
\Gamma(N \rightarrow P+\bar{l})=\frac{m_{N}}{32 \pi}\left[1-\left(\frac{m_{P}}{m_{N}}\right)^{2}\right]^{2}\left|\sum_{I} C^{I} W_{0}^{I}(0)\right|^{2},
$$

with the on-shell condition on the anti-lepton $-q^{2}=m_{l}^{2} \simeq 0$. Here $C^{I}$ are Wilson coefficients of the operator $\mathscr{O}^{I}$. Both $C^{I}$ and $\mathscr{O}^{I}$ need to be renormalized in the same scheme and scale. $W_{0}^{I}$ here is defined with the so renormalized $\mathscr{O}^{I}$. The renormalization constants calculated in the previous section are of the $\overline{\mathrm{MS}}$ at $\mu=2 \mathrm{GeV}$ and approximately unity.
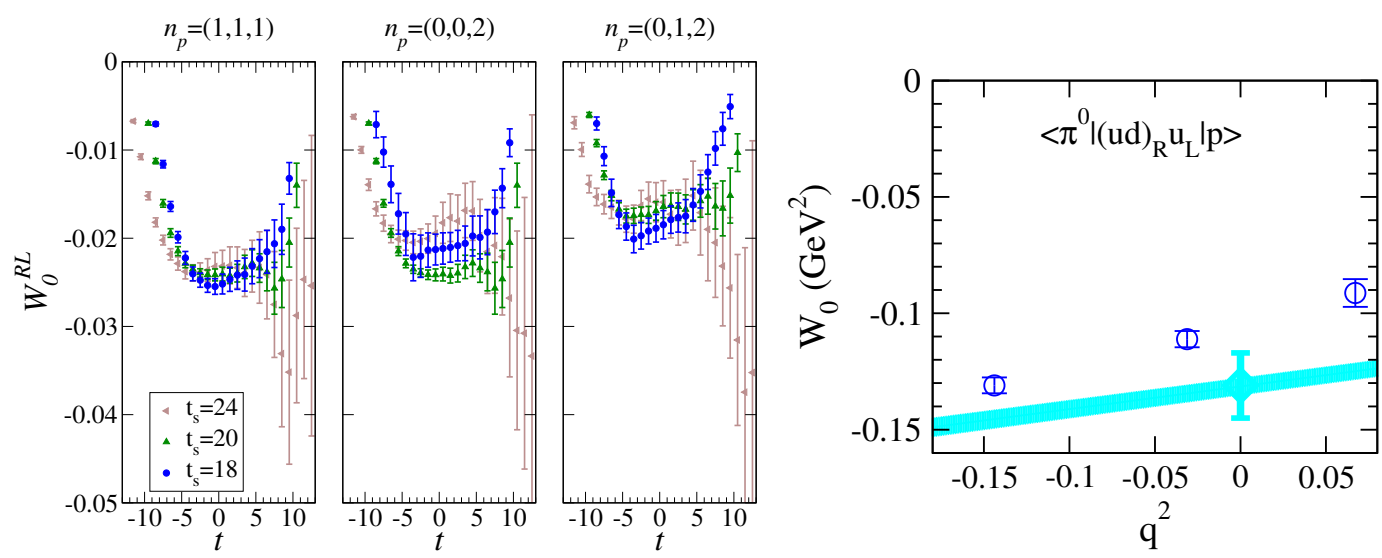

Figure 3: (a) (left panel) Relevant form factor of the $p \rightarrow \pi^{0}$ decay with $\mathscr{O}^{I}=\varepsilon_{i j k}\left(u^{i T} C P_{R} d^{j}\right) P_{L} u^{k}$ in lattice units as a function of operator position $t$. (b) (right panel) $W_{0}\left(q^{2}\right)$ for the same process (open symbol) compared against the chiral extrapolated results of domain-wall fermions shown as a cyan band [2].

We show here the bare matrix elements as preliminary results, which will not differ from those renormalized value in the errors. The left panel of Fig. 3 show the time-local definition of $W_{0}$ in lattice units for $p \rightarrow \pi^{0}$ with $\mathscr{O}^{I}=\varepsilon_{i j k}\left(u^{i T} C P_{R} d^{j}\right) P_{L} u^{k}$ where $i, j$ and $k$ are color indices. It is composed of three- and two-point functions with certain projections and of amplitudes of the two point functions. A linear combination with different projections are needed to finally disentangle $W_{0}$. Three different values of separation $t_{s}$ of proton source and pion sink are compared. In the 
figure $t$ is shifted so $t=0$ is always the central position between source and sink. Each of the three sub-panels show the result with fixed pion three momentum $\vec{p}=\frac{2 \pi}{L} \cdot \vec{n}_{p}$, where $L=64$. Taking plateaus at shortest separation $t_{s}=18$ and multiplying $a^{-2}$, one obtains the open symbol in the right panel plotted as a function of $q^{2}$. For a comparison, chiral extrapolated results of domain-wall fermions from the pion mass in the range of $340-700 \mathrm{MeV}$ [2] is shown as a cyan band. The filled symbol at $q^{2}=0$ indicates the final result of domain-wall fermions with the systematic error. Fitting and obtaining the $q^{2}=0$ value in our work will be necessary to get the final result. At this stage we only remark that a reasonable consistency between these two computations is observed. Assuming there is no large scaling violation persists in both studies, our new physical point result indicates there would not be a surprising chiral effect down to the physical pion mass.
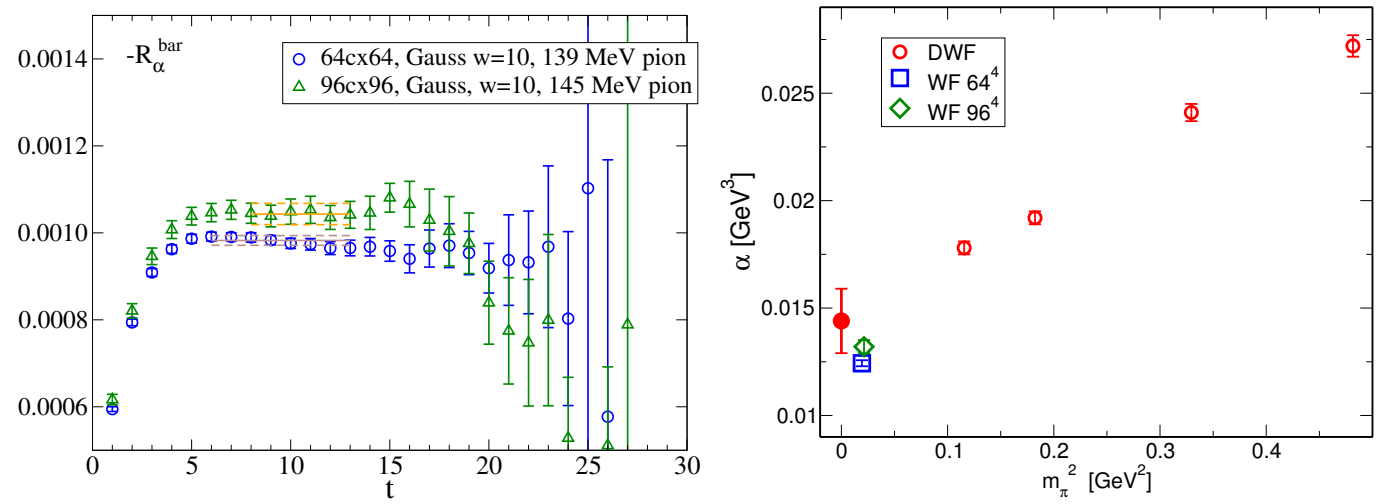

Figure 4: (a) (left panel) Low energy constant $\alpha$ calculated from the $p \rightarrow$ vacuum matrix element in lattice units as a function of operator position $t$. (b) (right panel) $\alpha$ in physical units plotted as a function of $m_{\pi}^{2}$ of the measured point (open symbols). Chiral limit of the domain wall fermions (red) [2] is shown as the filled symbol with the total systematic error.

Similar observation is found for the proton to vacuum matrix element, parameterized by the low energy constant $\alpha$,

$$
\left\langle 0\left|\varepsilon_{i j k}\left(u^{i T} C P_{R} d^{j}\right) P_{L} u^{k}\right| p\right\rangle=\alpha P_{L} u_{p},
$$

where $u_{p}$ is the proton spinor. To the leading order in the baryon chiral perturbation theory, the relevant form factor of $p \rightarrow \pi^{0}$ decay with the same operator is proportional to $\alpha$. The left panel of Fig. 4 is the $t$-local definition of $\alpha$ with the proton source at origin and the operator at $t$. Here not only $64^{4}$ but also $96^{4}$ lattices are used. Finite volume effects observed are about $6 \%$. The fitted value at plateau are plotted in the right panel at the corresponding $m_{\pi}^{2}$ point and compared against the chiral extrapolation of the domain-wall fermions [2].

\section{Summary and outlook}

Proton decay matrix elements were computed using improved Wilson fermion ensembles generated by the PACS collaboration. The use of the physical quark mass point as well as the RI/SMOM non-perturbative renormalization made it possible to completely remove the systematic uncertainty associated with the chiral extrapolation performed in the previous studies. The preliminary results indicate there is no strong non-analytic quark mass dependence on the relevant form 
factors near the physical point. We will extend this study with more statistics to access the remnant systematic effects, such as the source-sink separation. Furthermore, the complete set of the form factors for the Kaon final states will be computed. These will cover all of the most relavant channels of a pseudoscalr final state for any low energy theory of GUTs with three-quark operators.

\section{Acknowledgments}

Numerical calculations are performed on the supercomputer K at RIKEN Center for Computational Science (R-CCS), Hokusai BigWaterfall at RIKEN, Oakforest-PACS supercomputer operated by the Joint Center for Advanced High Performance Computing (JCAHPC). This work is supported in part by JSPS KAKENHI Grant Nos. 16K05320. N.T. is supported in part by the Joint Research Program of R-CCS.

\section{References}

[1] SUPER-KAMIOKANDE collaboration, Search for proton decay via $p \rightarrow e^{+} \pi^{0}$ and $p \rightarrow \mu^{+} \pi^{0}$ in 0.31 megaton-years exposure of the Super-Kamiokande water Cherenkov detector, Phys. Rev. D95 (2017) 012004 [1610.03597].

[2] Y. Aoki, T. Izubuchi, E. Shintani and A. Soni, Improved lattice computation of proton decay matrix elements, Phys. Rev. D96 (2017) 014506 [1705.01338].

[3] A. Martin and G. C. Stavenga, Non-perturbative proton stability, Phys.Rev. D85 (2012) 095010 [1110.2188].

[4] J.-S. Yoo, Y. Aoki, T. Izubuchi and S. Syritsyn, Proton decay matrix element on lattice at physical pion mass, PoS LATTICE2018 (2019) 187 [1812.09326].

[5] T. Blum, T. Izubuchi and E. Shintani, A new class of variance reduction techniques using lattice symmetries, Phys.Rev. D88 (2013) 094503 [1208.4349].

[6] E. Shintani, R. Arthur, T. Blum, T. Izubuchi, C. Jung and C. Lehner, Covariant approximation averaging, Phys. Rev. D91 (2015) 114511 [1402.0244].

[7] G. Martinelli, C. Pittori, C. T. Sachrajda, M. Testa and A. Vladikas, A General method for nonperturbative renormalization of lattice operators, Nucl. Phys. B445 (1995) 81 [hep-lat/9411010].

[8] C. Sturm et al., Renormalization of quark bilinear operators in a momentum- subtraction scheme with a nonexceptional subtraction point, Phys. Rev. D80 (2009) 014501 [0901.2599].

[9] RBC collaboration, Continuum Limit Physics from 2+1 Flavor Domain Wall QCD, Phys. Rev. D83 (2011) 074508 [1011.0892].

[10] Y. Aoki, C. Dawson, J. Noaki and A. Soni, Proton decay matrix elements with domain-wall fermions, Phys. Rev. D75 (2007) 014507 [hep-lat/ 0607002 ].

[11] E. Shintani, K.-I. Ishikawa, Y. Kuramashi, S. Sasaki and T. Yamazaki, Nucleon form factors and root-mean-square radii on a $(10.8 \mathrm{fm})^{4}$ lattice at the physical point, Phys. Rev. D99 (2019) 014510 [1811.07292].

[12] JLQCD collaboration, Nucleon decay matrix elements from lattice qcd, Phys. Rev. D62 (2000) 014506 [http://arXiv.org/abs/hep-lat/9911026]. 